\title{
Talking the Talk in Citizen Science
}

Mahboobeh Harandi

Syracuse University

Syracuse, NY 13210, USA

mharandi@syr.edu

\author{
Corey Brian Jackson \\ Syracuse University \\ Syracuse, NY 13210, USA \\ cjacks04@syr.edu
}

$\begin{array}{ll}\text { Carsten Osterlund } & \text { Kevin Crowston } \\ \text { Syracuse University } & \text { Syracuse University } \\ \text { Syracuse, NY 13210, USA } & \text { Syracuse, NY 13210, USA } \\ \text { costerlu@syr.edu } & \text { crowston@syr.edu }\end{array}$

Syracuse, NY 13210, USA

crowston@syr.edu

Permission to make digital or hard copies of part or all of this work for personal or classroom use is granted without fee provided that copies are not made or distributed for profit or commercial advantage and that copies bear this notice and the full citation on the first page. Copyrights for third-party components of this work must be honored. For all other uses, contact the owner/author(s).

CSCW'18 Companion, Nov. 2âASS7, 2018, Jersey City, NJ, USA.

Copyright is held by the author/owner(s).

ACM ISBN 978-1-4503-6018-0/18/11.

http://dx.doi.org/10.1145/3272973.3274084

\begin{abstract}
Increasingly, citizen scientists do work beyond the primary goal of the project coordinated via the discussion board. These activities often take place in discussion boards and have a set of terminologies norms for contributing. For newcomers, learning new terminologies presents a challenge since there are no formal opportunities for them to learn the terms and volunteers who join later need to learn more than volunteers who join earlier in a project life-cycle. In this poster, we examine how newcomers terminology uses shifts over the course of two citizen science projects. We find that, although, newcomers joining later might face obstacles, terminology use among newcomers associated with advanced work increase over the project's life-cycle. The analysis can help the science team assess whether newcomers on the talk page have either adopted advanced terminologies and if they need to have a more formal resource such as tutorial or blog posts.
\end{abstract}

\section{Author Keywords}

citizen science; newcomers; terminology adoption.

\section{Introduction}

Citizen science projects engage members of the public in scientific research by asking them to perform tasks such as image labeling or data collection [1]. For instance, in the Galaxy Zoo project volunteers review images of galaxies 
and categorize them based on their morphological characteristics (e.g., whether they are elliptical are spiral). These data are then used by astrophysicists to research astronomical phenomenon.

More recently, some citizen science projects ask volunteers to take on more advanced tasks such as article writing or supplying new labels to data [3]. Much of the "advanced work" is coordinated via conversations on the discussion boards [2]. To be effective contributors, newcomers need to become experts in the language of the community. However, several challenges exist in adopting new language. First, most projects only offer training in the primary work of the project e.g., how to make a classification. Second, over time language becomes increasingly complex, meaning newcomers need to apply more effort to identify and learn these terms. In this poster, we studied comments of newcomers in two citizen science projects to understand how terminology use change over time.

\section{Setting: Citizen Science}

To understand how newcomers adopt terminologies we studied the usage of different terminologies in two citizen science projects: Gravity Spy (http://gravityspy.org) and Snapshot Serengeti (https://www.snapshotserengeti.org). In Snapshot Serengeti, volunteers classifying images of animal species. Volunteers classify by applying labels from a list of sixty three species to images. In Gravity Spy [4], volunteers classify images of glitches (noise events) from the LIGO detectors using one of twenty labels. Examples of labels in both projects are listed in Table 1. In both projects, volunteers receive training on how to make a classification and are given definitions of the labels.

While both projects have a classification task, only Gravity Spy has an advanced task, in which volunteer are asked to find and name unknown classes of glitches. One way of identifying new classes of data is that volunteers add a new label for the glitch in a comment, which they can post after classifying the image. Coming up with new labels happens on the discussion boards and requires the use of terminology (which we call citizen terms) pointing to its morphological characteristics, e.g., descending or chirpish.

In this poster, we analyzed how newcomers used different terminologies related to the primary classification task and the advanced work task. Specifically, we address: What are the characteristics of terminology use and evolution in projects with different tasks?

\section{Data and Analysis}

We collected the comments volunteers posted in Snapshot Serengeti and Gravity Spy. Each record contains a user id, a timestamp, and the comment text. First, we parsed each comment into uni-grams. Since we are interested in how newcomers adopt different terminologies, we focused on terms which volunteers use in the primary classification task (called scientist labels) and terms we suspect are associated with advanced work - frequent terms. These are terms used, for example, to label and describe new glitch classes.

The frequent terms were derived by using term frequency inverse document frequency (tf-idf) scores. Tf-idf provides a univariate measurement of a terms importance in a corpus. We chose the first twenty of these high tf-idf terms to represent frequent domain terms. Examples of frequent terms in Gravity Spy and Snapshot Serengeti are in Table 1.

We calculated the normalized term frequency of scientist labels and frequent terms posted by each volunteer over the first month of commenting who have left comments for at least two days. We ran a linear model to understand how 


\begin{tabular}{lll}
\hline & \multicolumn{1}{c}{$\begin{array}{c}\text { scientist } \\
\text { labels }\end{array}$} & \multicolumn{1}{c}{$\begin{array}{c}\text { frequent } \\
\text { terms }\end{array}$} \\
\hline \multirow{2}{*}{ Gravity Spy } & $\begin{array}{l}\text { blip, whistle, chirp, } \\
\text { tomte, scratchy, helix }\end{array}$ & $\begin{array}{l}\text { hz, koi, possiblenewglitch, } \\
\text { Ifb, violin,1080 }\end{array}$ \\
\hline \multirow{2}{*}{ Snapshot Serengeti } & $\begin{array}{l}\text { aardvark, baboon, bat } \\
\text { hare, lion, kudu }\end{array}$ & $\begin{array}{l}\text { selfie, hippo, serengeti, } \\
\text { antelope, closeup }\end{array}$ \\
\hline
\end{tabular}

Table 1: Examples of scientist labels and frequent terms in Gravity Spy and Snapshot Serengeti

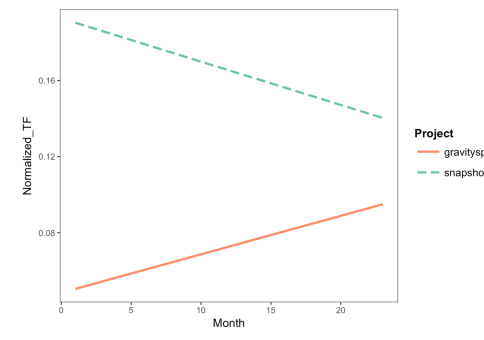

Figure 1: The interaction plot for frequency of scientist labels

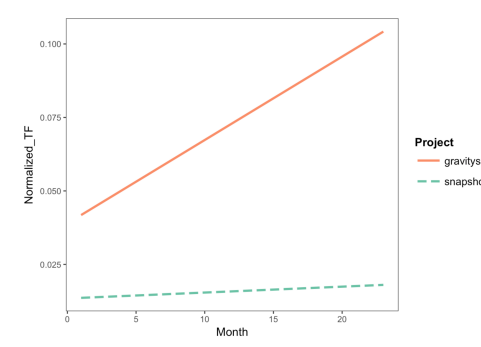

Figure 2: The interaction plot for frequency of frequent terms the project, the month that volunteers started commenting in each project and interaction between them affect the usage of scientist labels and frequent terms in their comments. Finally, we plotted the interaction between the project and the month over frequency of scientist labels and frequent terms.

\section{Results and Discussion}

There are 532 and 1,350 volunteers in Gravity Spy and Snapshot Serengeti, respectively, who posted 28,020 and 54,762 comments during their first month contributing to the fora.

Newcomers in Snapshot used significantly more scientist labels in their comments than the newcomers in Gravity Spy regardless of the projects' age. Since the number of scientist labels and newcomers in Snapshot is almost three times bigger than Gravity Spy, there is a significant difference in using scientist labels in their comments in Snapshot. A significant regression equation was found $(F(3,38)=13.88, p$ $<.0001$ ), with an adjusted $R^{2}$ of .48 (See Table 2 ). The frequency of scientist labels in Snapshot is 0.144 higher than Gravity Spy's. The project is the only significant predictor for the frequency of scientist labels. The interaction between the project and the month is plotted in Figure 1.
Newcomers behaviors in terms of using frequent terms is different than using scientist labels. Since volunteers in Snapshot are not required to find new species, they are not using frequent terms increasingly as the project is aging. However, newcomers in both projects use more frequent terms if they join the project later in compared to the ones who started commenting earlier in the project. A significant regression equation was found $(F(3,38)=26.23, p<.0001)$, with an adjusted $R^{2}$ of .65 (See Table 2). The frequency of frequent terms is increasing 0.003 terms in each month. But, as Snapshot project is aging, the frequency of frequent terms is decreasing 0.003 terms. The month and the interaction between the month and the project are significant predictors of frequent terms. The interaction between the project and the month is plotted in Figure 2. The frequency of frequent terms among newcomers' comments is increasing significantly over time unless we consider which project is aging.

\section{Conclusion}

In this study, we showed the different adoption characteristics of scientist labels and frequent terms depending on required tasks in a project. Our results showed volunteers adopt the advanced terminologies in the first month of commenting if it is part of the community norms despite lack of any formal resources to introduce relevant terminolo- 


\begin{tabular}{lllll} 
& Scientist Labels & $t$ & Frequent Terms & $t$ \\
\hline Month & $0.002(0.002)$ & 1.231 & $0.003^{* *}(0.001)$ & 3.560 \\
Project & $0.144^{* * *}(0.032)$ & 4.476 & $-0.026(0.016)$ & -1.633 \\
Month:SnapshotProject & $-0.004(0.002)$ & -1.784 & $-0.003^{*}(0.001)$ & -2.257 \\
R-squared & 0.48 & & 0.65 & \\
\hline${ }^{* * *} P<0001^{* *} p<001^{*} p<0.05$ & & &
\end{tabular}

${ }^{* * *} p<.0001,{ }^{* *} p<.001,{ }^{*} p<0.05$

Table 2: Linear model results for scientist labels and frequent terms

gies around the advanced tasks. However, they do not use these terms increasingly as the project is aging if the focus of the project is not finding new classes of data. The dif ferences outlined here point to the need for citizen science projects to develop strategies to socialize newcomers into the varied tasks within the community - classifying as well as talking. Additionally, given the unique science jargon developing across citizen science projects, this work highlights the need for additional studies pointing to the varied ways newcomers come to learn the language of a community.

\section{Acknowledgements}

We thank the volunteers, Zooniverse, and our Gravity Spy collaborators. This material is based on work supported by the National Science Foundation under Grant INSPIRE $15-47880$.

\section{REFERENCES}

1. R Bonney, H Ballard, R Jordan, E McCallie, T Phillips, J Shirk, and C C Wilderman. 2009. Public Participation in Scientific Research: Defining the Field and

Assessing Its Potential for Informal Science Education. Technical Report.

2. Carolin Cardamone, Kevin Schawinski, Marc Sarzi, Steven P Bamford, Nicola Bennert, C M Urry, Chris Lintott, William C Keel, John Parejko, Robert C Nichol, Daniel Thomas, Dan Andreescu, Phil Murray, Jordan
Raddick, Anže Slosar, Alex Szalay, and Jan Vandenberg. 2009. Galaxy Zoo Green Peas: discovery of a class of compact extremely star-forming galaxies. Monthly Notices of the Royal Astronomical Society 399, 3 (Nov. 2009), 1191-1205.

3. Corey Jackson, Kevin Crowston, Carsten Østerlund, and Mahboobeh Harandi. 2018. Folksonomies to Support Coordination and Coordination of Folksonomies. Computer Supported Cooperative Work (CSCW) (10 May 2018).

4. Michael Zevin, Scott Coughlin, Sara Bahaadini, Emre Besler, Neda Rohani, Sarah Allen, Miriam Cabero, Kevin Crowston, Aggelos Katsaggelos, Shane Larson, Tae Kyoung Lee, Chris Lintott, Tyson Littenberg, Andrew Lundgren, Carsten Øesterlund, Joshua Smith, Laura Trouille, and Vicky Kalogera. 2017. Gravity Spy: Integrating Advanced LIGO detector characterization, machine learning, and citizen science. Classical and Quantum Gravity 34, 6 (2017). 